Long-term bisphosphonate treatment of postmenopausal osteoporosis is associated with reduction of bone turnover and might, therefore, have deleterious adverse effects on the repair of microdamage and result in increased incidence of fracture.

Chapurlat et al. have performed a crosssectional study of transiliac bone biopsies from 50 osteoporotic women ( $\geq 5$ years postmenopausal) and 12 recently-dead control cadavers. All women enrolled in the study were taking pamidronate ( $90 \mathrm{mg}$, four times a year), alendronate $(10 \mathrm{mg}$ daily or $70 \mathrm{mg} /$ week) or risendronate ( $5 \mathrm{mg}$ daily or $35 \mathrm{mg} / \mathrm{week}$ ), and 48 of these participants had been taking their medication for $\geq 3$ years.

Bone remodeling was reduced in the osteoporotic women undergoing bisphosphonate treatment, with activation frequency of 0.06 $\pm 0.07 /$ year $($ mean $\pm S D$ ) and mean bone formation rate of $0.004 \mu \mathrm{m}^{3} / \mu \mathrm{m}^{2} /$ day. No difference in cancellous bone microcrack frequency was observed between the treated women and controls $\left(0.13\right.$ microcracks $/ \mathrm{mm}^{2}$ and 0.05 microcracks $/ \mathrm{mm}^{2}$, respectively, $P=0.59$ ) and no trabecular microcracks were visualized using green calcein stain for $54 \%$ of bone samples from treated women and $58 \%$ of control samples. No relationship between microcrack density and age, microcrack frequency and duration of bisphosphonate treatment or microcrack frequency and activation frequency was found in the 50 osteoporotic women evaluated.

The authors concluded that long-term bisphosphonate therapy for osteoporosis does not result in increased microdamage of the iliac bone in postmenopausal women, despite reduced bone remodeling.

Original article Chapurlat RD et al. (2007) Microcrack frequency and bone remodeling in postmenopausal osteoporotic women on long-term bisphosphonates: a bone biopsy study. J Bone Miner Res 22: 1502-1509

\section{HRT does not increase the risk of vascular events in postmenopausal women with SLE}

Hormone replacement therapy (HRT) is still indicated for the treatment of some postmenopausal women. Whether postmenopausal women with systemic lupus erythematosus (SLE) can safely use it is uncertain, because HRT might increase the occurrence of arterial and venous thrombotic events. Fernández et al. analyzed data from the LUMINA study - a multiethnic, longitudinal study of outcome in SLE patients - to assess the influence of HRT on the occurrence of thrombotic events in postmenopausal women.

The LUMINA cohort includes patients aged $\geq 16$ years with SLE disease duration $\leq 5$ years. Ten of the 82 postmenopausal women in this cohort (mean \pm SD age $53.7 \pm 7.7$ years) were excluded from analysis (9 were positive for antiphospholipid antibodies and 1 had suffered an arterial thrombotic event before HRT exposure). Of the remaining 72 patients, 40 were never-users and 32 were HRT users; data on dose and exact duration of exposure to HRT were not available, but overall in this group exposure duration was $60 \%$ of total SLE disease duration.

HRT use was not associated with an increased occurrence of thrombotic events. Indeed, there was a trend towards decreased occurrence of arterial events in HRT users in the univariate analyses, but this effect became nonsignificant after adjustment for confounders.

The findings indicate that HRT might be safe for postmenopausal women with SLE, as long as these patients are not at high-risk for thromboembolic events.

Original article Fernández M et al. (2007) Systemic lupus erythematosus in a multiethnic US cohort (LUMINA II): relationship between vascular events and the use of hormone replacement therapy in postmenopausal women. J Clin Rheumatol 13: 261-265

\section{Identification of two potential biomarkers indicating radiographic progression of hip OA}

Although several risk factors for osteoarthritis (OA) progression have been identified, such as age and obesity, no prognostic serum biomarkers have been defined. Lane and colleagues hypothesized that antagonists of the $W n t / \beta$-catenin signaling pathway, which is involved in skeletal and joint development, might be useful for predicting the course of OA. They studied whether the serum levels of two such Wnt signaling antagonists, FRP (Frizzled-related protein) and Dkk-1, can predict incidence and/or radiographic progression of hip OA.

The authors randomly selected 800 elderly women (age $\geq 65$ years) from a cohort of 5,928 subjects with two sets of hip radiographs 